\title{
ARTICLES
}

\section{In vivo staging of regional amyloid deposition}

\section{OPEN}

Michel J. Grothe, PhD

Henryk Barthel, MD

Jorge Sepulcre, MD, PhD

Martin Dyrba, PhD

Osama Sabri, MD

Stefan J. Teipel, MD

For the Alzheimer's

Disease Neuroimaging Initiative

Correspondence to

Dr. Grothe:

michel.grothe@dzne.de

Editorial, page 2029

Supplemental data at Neurology.org

\section{ABSTRACT}

Objectives: To estimate a regional progression pattern of amyloid deposition from cross-sectional amyloid-sensitive PET data and evaluate its potential for in vivo staging of an individual's amyloid pathology.

Methods: Multiregional analysis of florbetapir (18 F-AV45)-PET data was used to determine individual amyloid distribution profiles in a sample of 667 participants from the Alzheimer's Disease Neuroimaging Initiative cohort, including cognitively normal older individuals (CN) as well as patients with mild cognitive impairment and Alzheimer disease (AD) dementia. The frequency of regional amyloid positivity across $\mathrm{CN}$ individuals was used to construct a 4-stage model of progressing amyloid pathology, and individual distribution profiles were used to evaluate the consistency of this hierarchical stage model across the full cohort.

Results: According to a 4-stage model, amyloid deposition begins in temporobasal and frontomedial areas, and successively affects the remaining associative neocortex, primary sensory-motor areas and the medial temporal lobe, and finally the striatum. Amyloid deposition in these brain regions showed a highly consistent hierarchical nesting across participants, where only $2 \%$ exhibited distribution profiles that deviated from the staging scheme. The earliest in vivo amyloid stages were mostly missed by conventional dichotomous classification approaches based on global florbetapir-PET signal, but were associated with significantly reduced CSF A $\beta 42$ levels. Advanced in vivo amyloid stages were most frequent in patients with $A D$ and correlated with cognitive impairment in individuals without dementia.

Conclusions: The highly consistent regional hierarchy of PET-evidenced amyloid deposition across participants resembles neuropathologic observations and suggests a predictable regional sequence that may be used to stage an individual's progress of amyloid pathology in vivo. Neurology ${ }^{\circledR}$ 2017;89:2031-2038

\section{GLOSSARY}

$\mathbf{A D}=$ Alzheimer disease; $\mathbf{A D N I}=$ Alzheimer's Disease Neuroimaging Initiative; $\mathbf{C N}=$ cognitively normal; $\mathbf{D R}=$ delayed recall; $\mathbf{G M}=$ gray matter; $\mathbf{M C I}=$ mild cognitive impairment; $\mathbf{P V E}=$ partial volume effects; $\mathbf{S U V R} \mathbf{R}_{\mathbf{C e r}}=$ standard uptake value ratios scaled to the mean uptake of the whole cerebellum; TMT-B = Trail Making Test B.

A distinct regional progression pattern of cerebral amyloid deposits has been estimated from case series of neuropathologic examinations and forms the basis of widely used staging schemes for the characterization of an individual's extent of amyloid pathology at autopsy. ${ }^{1,2}$ Interestingly, early phases of amyloid deposition are mainly found in individuals who were cognitively unimpaired at last clinical evaluation, arguing for a protracted preclinical phase of amyloid pathology. ${ }^{2-7}$

From the German Center for Neurodegenerative Diseases (DZNE) (M.J.G., M.D., S.J.T.), Rostock; Department of Nuclear Medicine (H.B., O.S.), University of Leipzig, Germany; Gordon Center for Medical Imaging (J.S.), Division of Nuclear Medicine and Molecular Imaging, Department of Radiology, Massachusetts General Hospital and Harvard Medical School, Boston; Athinoula A. Martinos Center for Biomedical Imaging (J.S.), Charlestown, MA; and Department of Psychosomatic Medicine (S.J.T.), University of Rostock, Germany.

Data used in preparation of this article were obtained from the Alzheimer's Disease Neuroimaging Initiative (ADNI) database (adni.loni.usc.edu/). As such, the investigators within the ADNI contributed to the design and implementation of ADNI and/or provided data but did not participate in analysis or writing of this report. A complete listing of ADNI investigators can be found at Neurology.org.

Go to Neurology.org for full disclosures. Funding information and disclosures deemed relevant by the authors, if any, are provided at the end of the article. The Article Processing Charge was funded by the German Center for Neurodegenerative Diseases (DZNE).

This is an open access article distributed under the terms of the Creative Commons Attribution-NonCommercial-NoDerivatives License 4.0 (CC BY-NC-ND), which permits downloading and sharing the work provided it is properly cited. The work cannot be changed in any way or used commercially without permission from the journal. 
The advent of amyloid-sensitive PET imaging methods allows detecting cerebral amyloid deposits in living individuals with very high sensitivity and specificity. ${ }^{8-10}$ However, in contrast to the neuropathologic staging schemes, PET imaging data are mainly being used for the characterization of global amyloid burden into dichotomous positive/negative categories. Recent imaging-pathologic correlation studies found that amyloid positivity as conventionally defined usually corresponds to relatively advanced phases of amyloid pathology. ${ }^{11,12}$

We explored the feasibility of in vivo PETbased amyloid staging using a large sample of amyloid-sensitive florbetapir ( $\left.{ }^{18} \mathrm{~F}-\mathrm{AV} 45\right)$-PET data covering the full clinical spectrum of Alzheimer disease (AD), and adopting established neuropathologic approaches for determining regional staging models of amyloid pathology and other proteinopathies. ${ }^{1,2,13-16}$ Specifically, this includes estimation of a regional progression sequence based on the frequency of regional amyloid positivity across participants and evaluation of the regional hierarchy of amyloid deposition along this sequence across individual deposition profiles. The identified in vivo amyloid stages were characterized with respect to conventional dichotomous florbetapir-PET classifications, CSF A 342 levels, as well as clinical diagnosis and cognition.

METHODS Participants. All data used in the present study were obtained from the Alzheimer's Disease Neuroimaging Initiative (ADNI) (adni-info.org). The present study sample consisted of a total of 667 participants enrolled in the ADNI-GO and ADNI-2 cohorts, who had concurrent ${ }^{18} \mathrm{~F}-\mathrm{AV} 45$-PET and highresolution structural MRI scans available. The sample included
179 cognitively normal (CN) older individuals, 403 patients with mild cognitive impairment (MCI), and 85 patients with $\mathrm{AD}$ dementia. Detailed diagnostic criteria have been reported previously ${ }^{17,18}$ and are published on the ADNI website (adni.loni. usc.edu/methods/). Sample characteristics are summarized in table 1 .

Standard protocol approvals, registrations, and patient consents. Data collection and sharing in ADNI was approved by the institutional review board of each participating institution and written informed consent was obtained from all participants.

Imaging data. Acquisition and standardized preprocessing steps of the multicentric MRI and PET imaging data in ADNI have been reported previously ${ }^{19,20}$ and are described in detail on the ADNI website (adni.loni.usc.edu/methods/). Briefly, structural MRI data were acquired on 3T scanning platforms using T1weighted sagittal 3D magnetization-prepared rapid gradient echo sequences. Florbetapir-PET scans were acquired during a 50 - to 70 -minute interval following a $370 \mathrm{MBq}$ bolus injection of florbetapir $\left({ }^{18} \mathrm{~F}\right)$. All ADNI imaging data undergo standardized preprocessing steps aimed at increasing data uniformity across the multicenter scanner platforms.

Procedures for preprocessing of the imaging data have been described in detail previously. ${ }^{19,20}$ Briefly, florbetapir-PET scans were rigidly coregistered to the corresponding structural MRI scan, corrected for partial volume effects (PVE), ${ }^{21,22}$ and spatially normalized to an aging/AD-specific reference template using the registration measures from the corresponding MRI scans.

Regional florbetapir-PET uptake values were sampled from 52 brain regions defined in the Harvard-Oxford structural atlas, ${ }^{23}$ covering the whole cerebral cortex, as well as hippocampus, amygdala, striatum, and thalamus. The atlas labels were propagated to the customized template space using nonlinear image registration, and multiplied with a binary gray matter (GM) mask of the reference template thresholded at 50\% GM probability. ${ }^{20}$ Regional florbetapir-PET uptake means were converted to standard uptake value ratios by scaling to the mean uptake of the whole cerebellum $\left.\left(S_{U V R}\right)_{C e r}\right)$ in non-PVE-corrected data. 22,24

Individual regional amyloid deposition profiles, i.e., the presence or absence of amyloid pathology in each of the 52 brain regions, were determined based on a cutoff of regional SUVR $\mathrm{Cer}=0.92$. This cutoff lies in between the 2 most commonly used cutoffs of $\mathrm{SUVR}_{\mathrm{Cer}}=1.10$ and $\mathrm{SUVR}_{\mathrm{Cer}}=1.17$ for establishing amyloid positivity based on the global cortical signal ${ }^{10,25-27}$ in non-PVE-corrected florbetapir-PET data and was extrapolated to

\begin{tabular}{|c|c|c|c|}
\hline \multirow[t]{2}{*}{ Table 1} & & & \\
\hline & $\mathrm{CN}$ & $\mathrm{MCl}$ & $A D$ \\
\hline $\mathrm{n}$ & 179 & 403 & 85 \\
\hline Age, y & $73.8 \pm 6.5$ & $71.7 \pm 7.7$ & $75.6 \pm 8.3$ \\
\hline Sex, M/F & $88 / 91$ & 220/183 & $49 / 36$ \\
\hline Education, $y$ & $16.6 \pm 2.5$ & $16.1 \pm 2.7$ & $15.7 \pm 2.8$ \\
\hline MMSE, AU & $29.1 \pm 1.2$ & $28.1 \pm 1.7$ & $22.9 \pm 2.0$ \\
\hline Delayed recall, AU & $7.5 \pm 4.0$ & $5.0 \pm 4.2$ & $0.7 \pm 1.1$ \\
\hline Trail Making Test B, s & $80 \pm 39$ & $106 \pm 58$ & $194 \pm 85$ \\
\hline
\end{tabular}

Abbreviations: $\mathrm{AD}=$ Alzheimer disease; $\mathrm{CN}=$ cognitively normal; $\mathrm{MCl}=$ mild cognitive impairment; $\mathrm{MMSE}=\mathrm{Mini}-\mathrm{Mental}$ State Examination.

Average values are reported as mean $\pm \mathrm{SD}$. 
the PVE-corrected florbetapir-PET data of this study using linear regression. ${ }^{26}$ More detailed information on cutoff selection is provided in appendix e-1 at Neurology.org.

Data analysis. Determination of a model of regionally progressing amyloid pathology. In analogy to the approach used in previous neuropathologic staging studies, ${ }^{1,2,13,14}$ a model of regionally progressing amyloid pathology was estimated based on the frequency of regional amyloid positivity across participants, where the proportion of participants who exhibit amyloid pathology in a given brain region is used as an indicator of this region's temporal involvement in the course of spatially progressing amyloid pathology. This approach critically depends on a high regional variance in amyloid deposition across the examined participants. Thus, due to our focus on the characterization of early phases of amyloidosis, and given that amyloid load is believed to reach global levels before the emergence of cognitive impairments, ${ }^{28}$ we restricted the determination of the progression model to the sample of cognitively normal older individuals.

In order to operationalize the regionally detailed progression model into a manageable staging system, the 52 ranked anatomical regions were merged into 4 larger anatomical divisions (1-4) based on equally sized proportions of the observed range of involvement frequencies (illustrated in figures 1 and e-1). Additional analyses also explored regionally less or more detailed 3- or 5-stage models (appendix e-1).

In vivo staging of individual amyloid pathology. Regardless of diagnosis, individual amyloid deposition profiles were used to stage each individual's florbetapir-PET scan according to the estimated 4-stage model of regional amyloid progression. For this, an anatomical division was defined positive for amyloid pathology if at least $50 \%$ of the regions included in this division exhibited suprathreshold signal in the respective participant. Participants who only exhibited amyloid deposition in the first division were classified as being in stage I, and successive stages II-IV were characterized by additional involvement of anatomical divisions 2, 3, and 4, respectively. Participants who exhibited amyloid positivity in any division $>1$ without concurrent amyloid positivity in the previous divisions were classified as being nonstageable. A high proportion of stageable vs nonstageable participants is an indicator of the general validity of the hierarchical staging scheme. ${ }^{15,16}$ Given that this proportion may be biased in the $\mathrm{CN}$ group, because it is also used for determining the staging model in this study, we further conducted sensitivity analyses across independent training and test samples for this group (appendix e-1).

For comparison, florbetapir-PET scans were also conventionally classified as amyloid-positive or amyloid-negative based on the commonly used cutoffs of SUVR $\mathrm{Cer}=1.10$ and $\mathrm{SUVR}_{\mathrm{Cer}}$ $=1.17$, applied to the global composite SUVR $\mathrm{Cer}_{\text {values that are }}$ made available in the ADNI database (Jagust Lab, UC Berkley; adni.loni.usc.edu/methods/pet-analysis).

In vivo amyloid stages in relation to a CSF biomarker of amyloid pathology. Amyloid stages were studied in relation to CSF A 42 levels, which are well-known to decrease as cortical amyloid deposition increases. ${ }^{29,30}$ CSF measures of $A \beta 42$ from the same study time point were available for $90 \%(n=603)$ of the study sample. Methods for CSF biomarker quantification in the ADNI cohort are based on the xMAP Luminex platform and Innogenetics/Fujirebio AlzBio3 immunoassay kits and are described in detail elsewhere ${ }^{31}$ (adni.loni.usc.edu/methods/). Differences in CSF A 342 levels across increasing amyloid stages were assessed using Spearman correlation and Mann-Whitney $U$ tests between the first 2 amyloid stages and the group without any evidence of regional amyloid deposition (stage 0 ).

In vivo amyloid stages in relation to clinical diagnosis and cognition. Differences in the distribution of amyloid stages between clinical diagnoses were assessed using $\chi^{2}$ test. In addition, for each diagnostic group separately, we assessed associations between progressing amyloid stage and cognitive performance in tests of episodic memory (30-minute delayed recall $[D R]$ of the Rey Auditory Verbal Learning Test) and executive function (Trail Making Test B [TMT-B]) using Spearman correlations. For comparison, associations between increasing amyloid load and cognitive performance were also assessed using continuous global

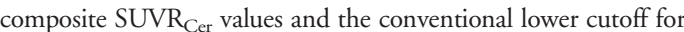

Figure 1 Model of regional amyloid progression and staging scheme
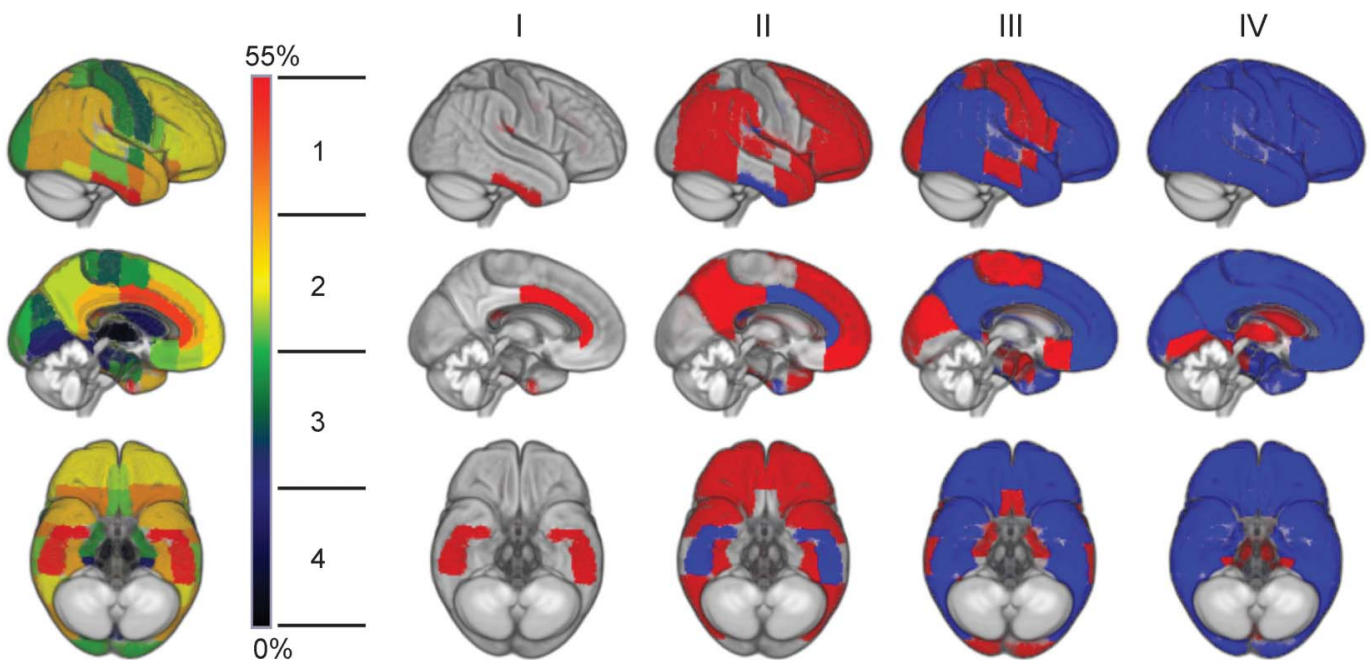

Brain renderings on the left illustrate the frequency of regional amyloid positivity across individuals on a color scale from black/blue (lowest) to yellow/red (highest). The 52 brain regions are merged into 4 larger anatomic divisions based on equal partitions of the frequency range (1-4). In the resulting 4-stage model of regional amyloid progression (I-IV), incremental stages are defined by involvement of higher numbered anatomic divisions (in red), in addition to the affected areas of the previous stage (blue). 
defining presence of detectable amyloid deposits $\left(\mathrm{SUVR}_{\mathrm{Cer}}>\right.$ 1.10).

RESULTS Model of regionally progressing amyloid pathology. The examined brain regions differed markedly with respect to the presence of amyloid pathology across $\mathrm{CN}$ individuals (figures 1 and e-1). Amyloid deposition was most frequently observed in the basal part of the temporal lobe (inferior temporal and fusiform gyrus), the anterior cingulate gyrus, and the parietal operculum ( $~ 55 \%-45 \%$ of individuals), followed by wide parts of the temporal, frontal, and

Figure 2 Staging of individual amyloid burden

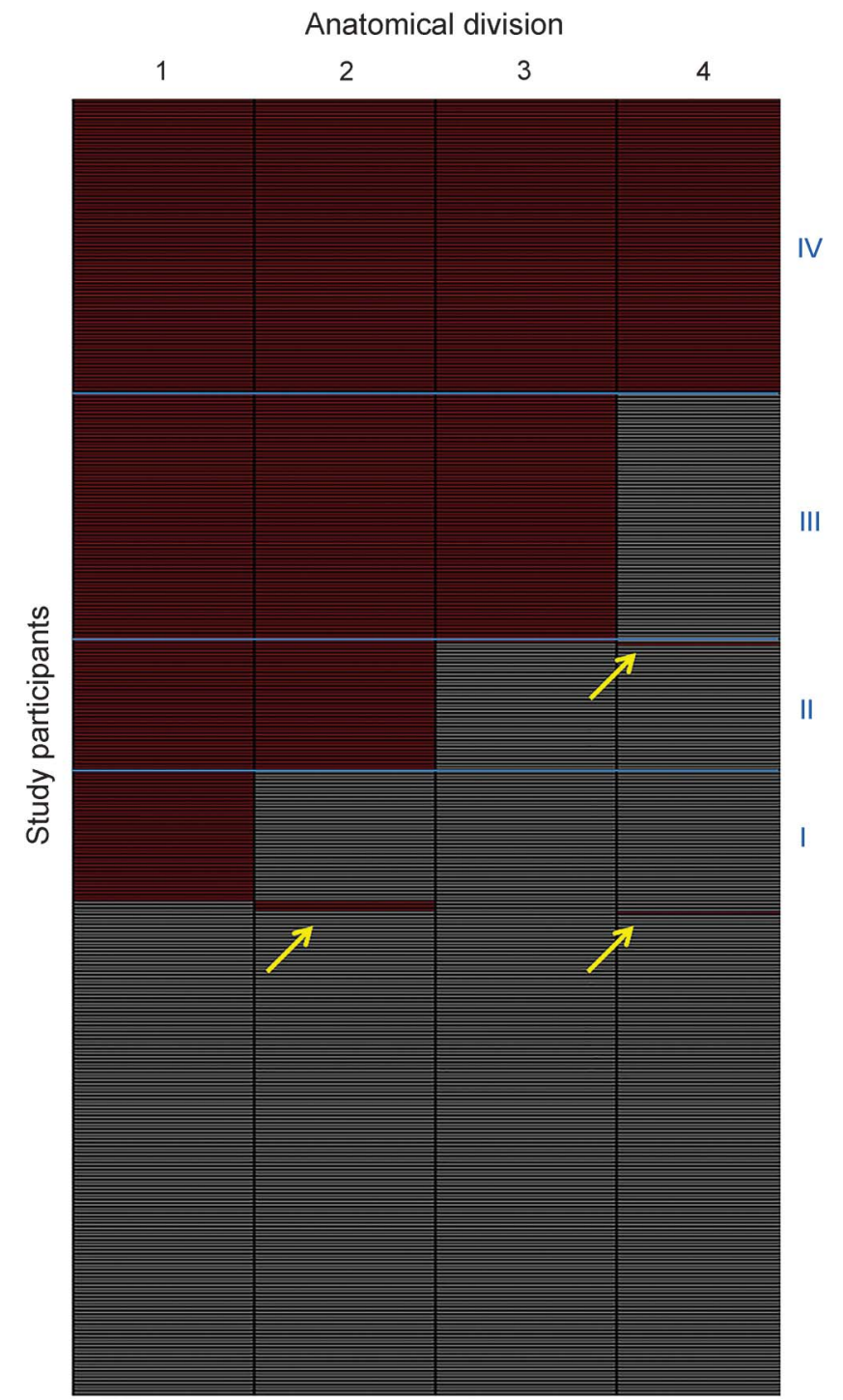

Each row of the matrix corresponds to a study participant and each column to one of the 4 anatomic divisions (1-4). Absence or presence of amyloid is denoted by gray and red, respectively. Amyloid deposition in the 4 anatomic divisions shows a consistent hierarchical nesting across participants, allowing us to stage $98 \%$ of the participants with detectable regional amyloid deposits into one of the 4 amyloid stages predicted by the regional progression model (blue boxes, I-IV). Only 8 participants showed distribution profiles that did not conform to the model (yellow arrows; 2 individuals [1 cognitively normal (CN), 1 Alzheimer disease] with deposition profiles of 1-1-0-1; 5 individuals [2 CN, 3 mild cognitive impairment $(\mathrm{MCl})]$ with 0-1-0-0; and 1 individual [MCl] with 0-0-0-1). parietal associative cortex ( 40\%-30\%). Amyloid deposits in primary sensory-motor cortices (precentral and postcentral, occipital pole, Heschl gyrus) and anterior medial temporal lobe structures were considerably less frequent $(\sim 25 \%-15 \%)$, and only a small minority showed amyloid positivity in the posterior medial temporal lobe and the striatum $(\sim 10 \%-5 \%)$. None of the participants exhibited amyloid positivity in the thalamus. This pattern was very robust against variations in the cutoff used for defining regional amyloid positivity (appendix e-1), and was used to define a 4-stage model of regional amyloid progression by grouping the brain regions from highest to lowest frequency into 4 anatomic divisions (figures 1 and e-1).

In vivo staging of individual amyloid pathology. Assessing regional amyloid deposition across the 4 anatomic divisions in individual florbetapir-PET scans yielded a highly consistent hierarchical deposition pattern across participants, allowing us to classify $98 \%$ (410/418) of participants with detectable regional amyloid deposition into any of the 4 successive amyloid stages (figures 2 and 3; table e-1). Sensitivity analyses across independent training and test samples indicated negligible bias in the proportion of stageable vs nonstageable participants in the $\mathrm{CN}$ group (appendix e-1). In vivo amyloid stages were positively associated with age in the $\mathrm{CN}(p=0.006)$ and MCI groups $(p=0.002)$, but not in the $\mathrm{AD}$ group $(p=$ $0.139)$ (appendix e-1).

Table 2 compares the in vivo amyloid staging scheme to conventional dichotomous classifications. Based on a global cortical cutoff of SUVR $\mathrm{Cer}=1.17$, all stage IV participants and the vast majority of stage III participants were identified as being amyloidpositive, whereas half of stage II and the vast majority of stage I participants were classified as being amyloid-negative. Even at a much lower cutoff of SUVR $_{\text {Cer }}=1.10,25 \%$ of stage II participants and the large majority of stage I participants (81\%) were still classified as amyloid-negative. However, characterizing the different amyloid stages in terms of an external CSF-based amyloid biomarker demonstrated a continuous decline in $A \beta 42$ levels across progressing amyloid stages (table 2; $\rho_{595}=-0.80, p<$ $0.001)$, which was already noticeable at the earliest amyloid stages I $(-7 \%, p=0.01)$ and II $(-29 \%$, $p<0.001)$ when compared to $A \beta 42$ levels of stage 0 .

Among participants exhibiting stageable amyloid deposition, distribution of in vivo amyloid stages differed significantly between diagnostic categories $\left(\chi^{2}=\right.$ 72.98, $p<0.001$; figure 3 and table e-1), with higher in vivo amyloid stages being more frequent in patients with $\mathrm{MCI}$ or $\mathrm{AD}$ compared to $\mathrm{CN}$ individuals. Amyloid stages III or IV (indicating extraneocortical involvement) 
Figure 3 Proportions of in vivo amyloid stages by clinical diagnosis

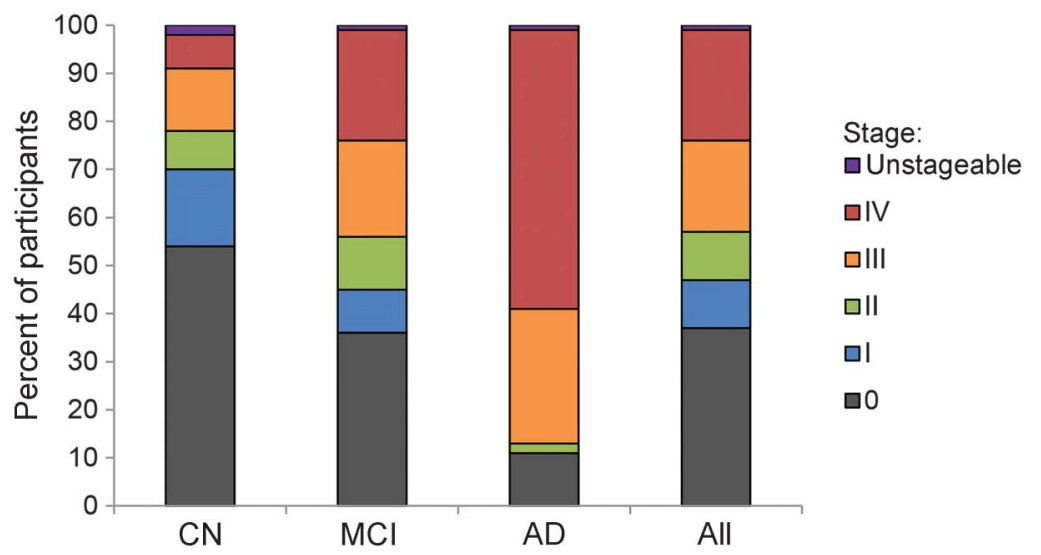

The relative proportions of in vivo amyloid stages were plotted for each diagnostic group separately and across all participants. Among participants exhibiting stageable amyloid deposition, there is a shift from lower to higher in vivo amyloid stages across the cognitively normal $(\mathrm{CN})$ over the mild cognitive impairment $(\mathrm{MCl})$ to the Alzheimer disease (AD) group. Unstageable cases were relatively rare across all diagnostic categories.

were observed in $96 \%$ of patients with $\mathrm{AD}, 66 \%$ of patients with $\mathrm{MCI}$, and $42 \%$ of $\mathrm{CN}$ individuals with detectable amyloid deposition. Within diagnostic groups separately, higher amyloid stages were associated with worse DR performance in $\mathrm{CN}\left(\rho_{79}=-0.40, p<\right.$ $0.001)$, and with worse DR $\left(\rho_{248}=-0.30, p<0.001\right)$ and TMT-B performance $\left(\rho_{245}=0.33, p<0.001\right)$ in $\mathrm{MCI}$, but did not associate with cognition in $\mathrm{AD}(p>$ $0.86)$ (table e-2). By contrast, among participants identified as being amyloid-positive by a conventional global cutoff of 1.10, SUVR $_{\text {Cer }}$ values correlated with cognition in MCI (DR: $\rho_{213}=-0.29, p<0.001$, TMT-B: $\left.\rho_{212}=0.35, p<0.001\right)$ and $\mathrm{AD}\left(\mathrm{DR}: \rho_{72}=-0.25\right.$, $p=0.035)$, but not in $\mathrm{CN}(p>0.17)$.

DISCUSSION In analogy to neuropathologic staging studies, we extrapolated a regional pattern of amyloid progression on the basis of florbetapir-PET amyloid deposition profiles across participants, and assessed its utility for in vivo staging of an individual's amyloid burden. The approach suggested a progression pattern from temporobasal and frontomedial areas, over the remaining associative neocortex, to primary sensory-motor cortex, the medial temporal lobe, and finally the striatum. Individual deposition profiles showed a highly consistent hierarchical nesting across these brain regions, arguing for the general validity of the progression model and enabling an in vivo staging of an individual's progress of amyloid pathology on the basis of cross-sectional imaging data.

The estimated pattern of regional amyloid progression in the florbetapir-PET data shows a remarkable resemblance with previous neuropathologic estimates. Particularly the progression from the associative neocortex over primary sensory-motor areas and the medial temporal lobe to the striatum is well-described in the neuropathologic literature and corresponds to phases 1 to 3 of the widely used Thal scheme of 5 progressive phases of cerebral amyloidosis. ${ }^{2,4}$ While the first Thal phase encompasses the whole associative neocortex without further regional distinction, previous neuropathologic studies specifically implicated basal portions of the temporal and frontomedial cortex in the initial phase of amyloid accumulation (corresponding to the first stage of the Braak A-B-C scheme of amyloid progression). ${ }^{1,3,5,6}$ However, other crosssectional ${ }^{11} \mathrm{C}$-PiB-PET estimates based on regional correlates of increasing global SUVR values pointed to medial frontal, medial parietal, and lateral temporoparietal areas as the sites of initial amyloid accumulation, without noticeable involvement of the inferior temporal cortex. ${ }^{12,32}$ Besides possible radiotracerrelated differences, these discrepancies are likely to be attributable to the different analytic approaches. A recent ${ }^{18} \mathrm{~F}$-florbetaben-PET study used a similar approach to the one employed in our study and reported a very similar pattern of most frequently observed amyloid deposits in basal temporal and frontomedial cortices, and least frequent deposits in the medial temporal lobe (the striatum was not investigated). ${ }^{33}$ However, differences in involvement frequencies across neocortical regions were rather small, which probably relates to a high proportion of participants with globally progressed amyloid

\begin{tabular}{|c|c|c|c|c|c|c|}
\hline \multirow[t]{2}{*}{ Table 2} & \multicolumn{6}{|c|}{ Amyloid stages in comparison to dichotomous classifications and CSF amyloid } \\
\hline & & 0 & 1 & II & III & IV \\
\hline $\mathrm{n}$ & & 249 & 68 & 63 & 127 & 152 \\
\hline SUVR $_{\text {Cer }}>$ & 1.17 & $1(<1 \%)$ & $3(4 \%)$ & 33 (52\%) & $118(93 \%)$ & $152(100 \%)$ \\
\hline SUVR $_{\text {Cer }}>$ & 1.10 & $6(2 \%)$ & $13(19 \%)$ & $47(75 \%)$ & $123(97 \%)$ & $152(100 \%)$ \\
\hline CSF A $42^{a}$ & $($ mean $\pm S D)$ & $224 \pm 36$ & $209 \pm 39^{b}$ & $160 \pm 39^{c}$ & $138 \pm 24^{c}$ & $127 \pm 20^{c}$ \\
\hline
\end{tabular}

Abbreviations: CSF A $\beta 42=$ CSF levels of $A \beta 42(\mathrm{pg} / \mathrm{mL}) ; \mathrm{SUVR}_{\mathrm{Cer}}=$ standard uptake value ratio with whole cerebellar reference region.

${ }^{\text {a }}$ CSF biomarker values were only available for a subset of participants ( $n=597$ ).

b Significantly different from stage 0 at $p<0.05$.

${ }^{c}$ Significantly different from stage 0 at $p<0.001$. 
pathology in the clinical sample used for model determination. Accordingly, no attempts were made in this previous study to use the estimated progression pattern in the context of an in vivo staging scheme.

In our individual staging analyses, only $2 \%$ of participants with detectable amyloid deposition violated the proposed hierarchy of regional involvement (figures 2 and 3 and table e-1). The consistent regional hierarchy of amyloid deposition in this large series of florbetapir-PET scans across the full clinical spectrum of AD strongly indicates that PET-measured amyloid deposition follows a predictable regional sequence that can be used analogously to established neuropathologic approaches for staging an individual's pathologic state along this sequence. However, in this cross-sectional data it remains unknown whether individuals inevitably progress from earlier to later stages, as well as under which circumstances and at which rates this progression occurs. A distinct advantage of the presented in vivo staging approach is that it will allow studying these questions through longitudinal amyloid-PET assessments and analysis of such data is warranted.

The earliest in vivo amyloid stages identify participants with regionally restricted amyloid deposits in specific neocortical association areas that are probably entirely missed in clinical routine binary visual assessment, ${ }^{11}$ and mostly missed by semiquantitative classification approaches based on suprathreshold global cortical signal, even at a relatively lenient cutoff (table 2). Decreased CSF A 442 levels compared to the stage 0 group corroborate the neurobiologic relevance of the regionally increased florbetapir-PET signal in these participants. Of course, the consistency of hierarchical amyloid deposition across participants has its limitation with respect to regional detail. Thus, while regionally more fine-grained staging schemes may potentially further increase the sensitivity to detect earliest focal amyloid deposits, they will also generally result in higher numbers of participants who show deviating deposition patterns (i.e., are nonstageable; appendix e-1). Moreover, the distinct amyloid progression pattern as estimated here and in neuropathologic case series ${ }^{1,2,34}$ may be different in genetically determined forms of amyloid accumulation, particularly with respect to the occurrence of striatal vs cortical amyloid. ${ }^{35-38}$ Different staging schemes may have to be developed for these populations.

Based on clinicopathologic correlations between striatal amyloid deposition (corresponding to Thal phase 3) and the occurrence of dementia, ${ }^{2,4,34}$ it has been suggested to incorporate an a priori distinction between cortical and striatal amyloid deposition in the analysis of amyloid-sensitive PET data, representing a pathologic 2-stage model that would possibly be more predictive of clinical status. ${ }^{39,40}$ In our data- driven estimation of an in vivo staging scheme, we provide evidence that (1) PET-measured striatal amyloid deposition (anatomical division 4 in our model) is extremely unlikely to occur without concomitant cortical amyloid (figure 2) and that (2) advanced in vivo amyloid stages are most frequently observed in patients with $\mathrm{AD}$ and associate with cognitive impairments in individuals without dementia. Comparison with conventional continuous measures of global amyloid load indicate that these show a higher dynamic range in clinically overt disease stages, whereas the presented in vivo staging approach may be particularly useful for stratification of early, preclinical stages of $\mathrm{AD}$.

A limitation of our in vivo imaging approach to amyloid staging is that the presence of regional amyloid necessarily needs to be defined by a signal cutoff that aims to separate noise from specific tracer binding. While we oriented this cutoff on most commonly used cutoffs for defining amyloid positivity on the basis of global cortical florbetapir-PET SUVR ${ }_{\text {Cer, }}$, cutoff definition is an arbitrary undertaking per se, and the transferability of global signal cutoffs to regional signals remains largely unexplored. Furthermore, in this study we used a constant regional cutoff, whereas it may be argued that differential cutoffs should be used due to differing noise levels between brain regions. ${ }^{33,35,40}$ Although the invariance of the regional amyloid pattern under varying cutoffs (appendix e-1) indicates little interregional variability in noise levels in our PVE-corrected data, the choice of cutoff definition might well affect the regional staging scheme and future research should investigate possible advantages of region-specific cutoffs for the presented in vivo amyloid staging approach. Moreover, as is the case for cutoffs applied to the global signal, ${ }^{12}$ the height of the regional cutoff affects the absolute numbers of individuals with detectable amyloid deposits. Thus, cutoff selection as well as other methodical choices, such as the regional detail of the staging scheme, should weigh off priorities for sensitivity or specificity depending on the actual research context or clinical application of the staging approach.

Pending replication of our findings in independent cohorts and using different radiotracers, the presented in vivo amyloid staging approach may become a valuable tool for characterizing an individual's amyloid burden for diagnostic purposes or for sample stratification in clinical trials.

\section{AUTHOR CONTRIBUTIONS}

Michel J. Grothe: study concept, analysis and interpretation of data, drafting/revising the manuscript for content. Henryk Barthel: study concept, interpretation of data, drafting/revising the manuscript for content. Jorge Sepulcre: study concept, contribution of analytic tools, drafting/revising the manuscript for content. Martin Dyrba: analysis of data, contribution of analytic tools. Osama Sabri: interpretation of data, drafting/revising the manuscript for content. 
Stefan Teipel: interpretation of data, drafting/revising the manuscript for content.

\section{STUDY FUNDING}

Data collection and sharing for this project was funded by the Alzheimer's Disease Neuroimaging Initiative (ADNI) (NIH grant U01 AG024904 and DOD ADNI [Department of Defense award number W81XWH-12-2-0012]). The ADNI was launched in 2003 by the National Institute on Aging (NIA), the National Institute of Biomedical Imaging and Bioengineering (NIBIB), the Food and Drug Administration, private pharmaceutical companies and nonprofit organizations, as a $\$ 60$ million, 5-year public-private partnership. ADNI is funded by the NIA, the NIBIB, and through contributions from the following: Alzheimer's Association; Alzheimer's Drug Discovery Foundation; Araclon Biotech; BioClinica, Inc.; Biogen Idec Inc.; Bristol-Myers Squibb Company; CereSpir, Inc.; Eisai Inc.; Elan Pharmaceuticals, Inc.; Eli Lilly and Company; EuroImmun; F. Hoffmann-La Roche Ltd. and its affiliated company Genentech, Inc.; Fujirebio; GE Healthcare; IXICO Ltd.; Janssen Alzheimer Immunotherapy Research \& Development, LLC.; Johnson \& Johnson Pharmaceutical Research \& Development LLC.; Lumosity; Lundbeck; Merck \& Co., Inc.; Meso Scale Diagnostics, LLC.; NeuroRx Research; Neurotrack Technologies; Novartis Pharmaceuticals Corporation; Pfizer Inc.; Piramal Imaging; Servier; Takeda Pharmaceutical Company; and Transition Therapeutics. The Canadian Institutes of Health Research is providing funds to support ADNI clinical sites in Canada. Private sector contributions are facilitated by the Foundation for the NIH (fnih.org). The grantee organization is the Northern California Institute for Research and Education, and the study is coordinated by the Alzheimer's Disease Cooperative Study at the University of California, San Diego. ADNI data are disseminated by the Laboratory for Neuroimaging at the University of Southern California.

\section{DISCLOSURE}

M. Grothe reports no disclosures relevant to the manuscript. H. Barthel received consultant and speaker honoraria as well as travel expenses from Piramal Imaging. J. Sepulcre is supported by the NIH (1K23EB019023). M. Dyrba reports no disclosures relevant to the manuscript. O. Sabri received consultant and speaker honoraria as well as travel expenses from Piramal Imaging. S. Teipel reports no disclosures relevant to the manuscript. Go to Neurology.org for full disclosures.

Received January 17, 2017. Accepted in final form August 14, 2017.

\section{REFERENCES}

1. Braak H, Braak E. Neuropathological stageing of Alzheimer-related changes. Acta Neuropathol 1991;82: 239-259.

2. Thal DR, Rub U, Orantes M, Braak H. Phases of A betadeposition in the human brain and its relevance for the development of AD. Neurology 2002;58:1791-1800.

3. Price JL, Morris JC. Tangles and plaques in nondemented aging and "preclinical" Alzheimer's disease. Ann Neurol 1999;45:358-368.

4. Murray ME, Lowe VJ, Graff-Radford NR, et al. Clinicopathologic and ${ }^{11} \mathrm{C}$-Pittsburgh compound $\mathrm{B}$ implications of Thal amyloid phase across the Alzheimer's disease spectrum. Brain 2015;138:1370-1381.

5. Price JL, Davis PB, Morris JC, White DL. The distribution of tangles, plaques and related immunohistochemical markers in healthy aging and Alzheimer's disease. Neurobiol Aging 1991;12:295-312.

6. Giannakopoulos P, Hof PR, Vallet PG, Giannakopoulos AS, Charnay Y, Bouras C. Quantitative analysis of neuropathologic changes in the cerebral cortex of centenarians. Prog Neuropsychopharmacol Biol Psychiatry 1995;19: 577-592.

7. Bennett DA, Wilson RS, Boyle PA, Buchman AS, Schneider JA. Relation of neuropathology to cognition in persons without cognitive impairment. Ann Neurol 2012;72:599-609.

8. Teipel S, Drzezga A, Grothe MJ, et al. Multimodal imaging in Alzheimer's disease: validity and usefulness for early detection. Lancet Neurol 2015;14:1037-1053.

9. Sabri O, Sabbagh MN, Seibyl J, et al. Florbetaben PET imaging to detect amyloid beta plaques in Alzheimer disease: phase 3 study. Alzheimers Dement 2015;11:964974.

10. Clark CM, Schneider JA, Bedell BJ, et al. Use of florbetapir-PET for imaging beta-amyloid pathology. JAMA 2011;305:275-283.

11. Thal DR, Beach TG, Zanette M, et al. [(18)F]flutemetamol amyloid positron emission tomography in preclinical and symptomatic Alzheimer's disease: specific detection of advanced phases of amyloid-beta pathology. Alzheimers Dement 2015;11:975-985.

12. Villeneuve S, Rabinovici GD, Cohn-Sheehy BI, et al. Existing Pittsburgh compound-B positron emission tomography thresholds are too high: statistical and pathological evaluation. Brain 2015;138:2020-2033.

13. Braak H, Del Tredici K, Rub U, de Vos RA, Jansen Steur EN, Braak E. Staging of brain pathology related to sporadic Parkinson's disease. Neurobiol Aging 2003;24:197211.

14. Josephs KA, Murray ME, Whitwell JL, et al. Updated TDP-43 in Alzheimer's disease staging scheme. Acta Neuropathol 2016;131:571-585.

15. Gertz HJ, Xuereb J, Huppert F, et al. Examination of the validity of the hierarchical model of neuropathological staging in normal aging and Alzheimer's disease. Acta Neuropathol 1998;95:154-158.

16. Jellinger KA. A critical reappraisal of current staging of Lewy-related pathology in human brain. Acta Neuropathol 2008;116:1-16.

17. Grothe MJ, Teipel SJ. Spatial patterns of atrophy, hypometabolism, and amyloid deposition in Alzheimer's disease correspond to dissociable functional brain networks. Hum Brain Mapp 2016;37:35-53.

18. Grothe MJ, Heinsen H, Amaro E Jr, Grinberg LT, Teipel SJ. Cognitive correlates of basal forebrain atrophy and associated cortical hypometabolism in mild cognitive impairment. Cereb Cortex 2016;26:2411-2426.

19. Teipel S, Grothe MJ. Does posterior cingulate hypometabolism result from disconnection or local pathology across preclinical and clinical stages of Alzheimer's disease? Eur J Nucl Med Mol Imaging 2016;43:526-536.

20. Teipel SJ, Kurth J, Krause B, Grothe MJ. The relative importance of imaging markers for the prediction of Alzheimer's disease dementia in mild cognitive impairment: beyond classical regression. Neuroimage Clin 2015;8:583-593.

21. Muller-Gartner HW, Links JM, Prince JL, et al. Measurement of radiotracer concentration in brain gray matter using positron emission tomography: MRI-based correction for partial volume effects. J Cereb Blood Flow Metab 1992;12:571-583.

22. Gonzalez-Escamilla G, Lange C, Teipel S, Buchert R, Grothe MJ. PETPVE12: an SPM toolbox for partial volume effects correction in brain PET: application to amyloid imaging with AV45-PET. Neuroimage 2017;147: 669-677.

23. Desikan RS, Segonne F, Fischl B, et al. An automated labeling system for subdividing the human cerebral cortex 
on MRI scans into gyral based regions of interest. Neuroimage 2006;31:968-980.

24. Catafau AM, Bullich S, Seibyl JP, et al. Cerebellar amyloid-beta plaques: how frequent are they, and do they influence 18F-florbetaben SUV ratios? J Nucl Med 2016; 57:1740-1745.

25. Joshi AD, Pontecorvo MJ, Clark CM, et al. Performance characteristics of amyloid PET with florbetapir F 18 in patients with Alzheimer's disease and cognitively normal subjects. J Nucl Med 2012;53:378-384.

26. Landau SM, Breault C, Joshi AD, et al. Amyloid-beta imaging with Pittsburgh compound B and florbetapir: comparing radiotracers and quantification methods. J Nucl Med 2013;54:70-77.

27. Fleisher AS, Chen K, Liu X, et al. Using positron emission tomography and florbetapir F18 to image cortical amyloid in patients with mild cognitive impairment or dementia due to Alzheimer disease. Arch Neurol 2011;68:1404-1411.

28. Jack CR Jr, Knopman DS, Jagust WJ, et al. Hypothetical model of dynamic biomarkers of the Alzheimer's pathological cascade. Lancet Neurol 2010;9:119-128.

29. Landau SM, Lu M, Joshi AD, et al. Comparing positron emission tomography imaging and cerebrospinal fluid measurements of beta-amyloid. Ann Neurol 2013;74: 826-836.

30. Grimmer T, Riemenschneider M, Forstl H, et al. Beta amyloid in Alzheimer's disease: increased deposition in brain is reflected in reduced concentration in cerebrospinal fluid. Biol Psychiatry 2009;65:927-934

31. Shaw LM, Vanderstichele H, Knapik-Czajka M, et al. Cerebrospinal fluid biomarker signature in Alzheimer's disease neuroimaging initiative subjects. Ann Neurol 2009;65:403-413.
32. Vlassenko AG, Benzinger TL, Morris JC. PET amyloidbeta imaging in preclinical Alzheimer's disease. Biochim Biophys Acta 2012;1822:370-379.

33. Cho H, Choi JY, Hwang MS, et al. In vivo cortical spreading pattern of tau and amyloid in the Alzheimer disease spectrum. Ann Neurol 2016;80:247-258.

34. Beach TG, Sue LI, Walker DG, et al. Striatal amyloid plaque density predicts Braak neurofibrillary stage and clinicopathological Alzheimer's disease: implications for amyloid imaging. J Alzheimers Dis 2012;28:869876.

35. Annus T, Wilson LR, Hong YT, et al. The pattern of amyloid accumulation in the brains of adults with Down syndrome. Alzheimers Dement 2016;12:538-545.

36. Klunk WE, Price JC, Mathis CA, et al. Amyloid deposition begins in the striatum of presenilin- 1 mutation carriers from two unrelated pedigrees. J Neurosci 2007;27: 6174-6184.

37. Koivunen J, Verkkoniemi A, Aalto S, et al. PET amyloid ligand $\left[{ }^{11} \mathrm{C}\right] \mathrm{PIB}$ uptake shows predominantly striatal increase in variant Alzheimer's disease. Brain 2008;131: 1845-1853.

38. Villemagne VL, Ataka S, Mizuno T, et al. High striatal amyloid beta-peptide deposition across different autosomal Alzheimer disease mutation types. Arch Neurol 2009;66: $1537-1544$

39. Beach TG, Thal DR, Zanette M, Smith A, Buckley C. Detection of striatal amyloid plaques with [18F]flutemetamol: validation with postmortem histopathology. J Alzheimers Dis 2016;52:863-873.

40. Shah N, Frey KA, Muller ML, et al. Striatal and cortical beta-amyloidopathy and cognition in Parkinson's disease. Mov Disord 2016;31:111-117.

\section{Visit the Neurology ${ }^{\circledR}$ Resident \& Fellow Website}

Click on Residents \& Fellows tab at Neurology.org.

Now offering:

- Neurology ${ }^{\circledR}$ Resident \& Fellow Editorial team information

- "Search by subcategory" option

- E-pearl of the Week

- RSS Feeds

- Direct links to Continuum ${ }^{\circledR}$, Career Planning, and AAN Resident \& Fellow pages

- Recently published Resident \& Fellow articles

- Podcast descriptions

(1) Find Neurolog $y^{\circledR}$ Residents \& Fellows Section on Facebook: http://tinyurl.com/o8ahsys

Follow Neurology ${ }^{\circledR}$ on Twitter: http://twitter.com/GreenJournal 


\section{Neurology}

In vivo staging of regional amyloid deposition

Michel J. Grothe, Henryk Barthel, Jorge Sepulcre, et al.

Neurology 2017;89;2031-2038 Published Online before print October 18, 2017

DOI 10.1212/WNL.0000000000004643

This information is current as of October 18, 2017

\section{Updated Information \&}

Services

Supplementary Material

References

\section{Citations}

Subspecialty Collections

Permissions \& Licensing

Reprints including high resolution figures, can be found at:

http://n.neurology.org/content/89/20/2031.full

Supplementary material can be found at:

http://n.neurology.org/content/suppl/2017/10/18/WNL.0000000000004643.DC1 http://n.neurology.org/content/suppl/2017/10/18/WNL.0000000000004643.DC2 http://n.neurology.org/content/suppl/2017/10/18/WNL.0000000000004643.DC3

This article cites 40 articles, 5 of which you can access for free at: http://n.neurology.org/content/89/20/2031.full\#ref-list-1

This article has been cited by 9 HighWire-hosted articles: http://n.neurology.org/content/89/20/2031.full\#\#otherarticles

This article, along with others on similar topics, appears in the following collection(s): Alzheimer's disease

http://n.neurology.org/cgi/collection/alzheimers_disease

PET

http://n.neurology.org/cgi/collection/pet

Information about reproducing this article in parts (figures,tables) or in its entirety can be found online at:

http://www.neurology.org/about/about_the_journal\#permissions

Information about ordering reprints can be found online:

http://n.neurology.org/subscribers/advertise

Neurology $®$ is the official journal of the American Academy of Neurology. Published continuously since 1951, it is now a weekly with 48 issues per year. Copyright Copyright (C) 2017 The Author(s). Published by Wolters Kluwer Health, Inc. on behalf of the American Academy of Neurology.. All rights reserved. Print ISSN: 0028-3878. Online ISSN: 1526-632X.

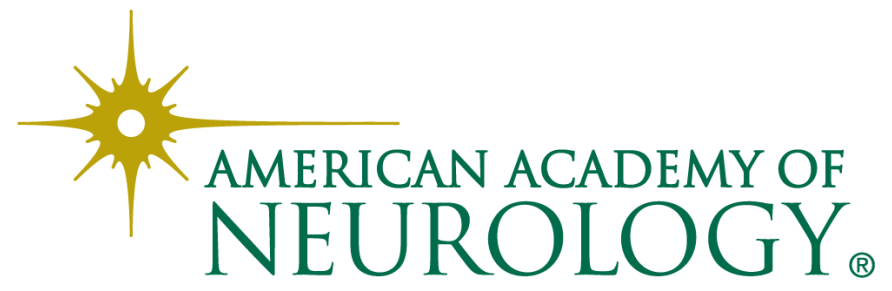

